QUADERNS DE FILOSOFIA VOL. VIII NÚM. 2 (202 I): 67-76

eISSN: $234 \mathrm{I}-3042$ DOI: I O.7203/QFIA. 8.2.207I I

Maria Medina-Vicent

Universitat Jaume I

\title{
Breve aproximación a los discursos gerenciales desde la perspectiva feminista ${ }^{1}$
}

Brief approach to managerial discourses from a feminist perspective

Resumen: En este escrito se presenta un resumen de la obra Mujeres y discursos gerenciales. Hacia la autogestión feminista (2020), donde se abordan desde una perspectiva feminista los discursos gerenciales dirigidos a las mujeres, desvelando la falsa neutralidad de sus premisas y la perpetuación de modelos de género dicotómicos en el seno de la gestión. Al mismo tiempo, se apunta a la actual mercantilización de las identidades y del movimiento por la igualdad, señalando los peligrosos virajes discursivos a los que se está sometiendo a dicho movimiento en la actualidad.

Abstract: In this article we present a summary of the work Mujeres y discursos gerenciales. Hacia la autogestión feminista (2020), where management discourses aimed at women are approached from a feminist perspective, revealing the false neutrality of their premises and the perpetuation of dichotomous gender models within management. At the same time, it points to the current commercialization of identities and the movement for equality, pointing out the dangerous discursive shifts that this movement is currently undergoing.

Palabras clave: discursos gerenciales, teoría feminista, liderazgo, despolitización.

Keywords: managerial discourses, feminist theory, leadership, depoliticization.

${ }^{1}$ Este trabajo se enmarca en el contexto del proyecto de investigación del Ministerio de Ciencia, Innovación y Universidades, con referencia PGC2018-097200-B-I00; y del proyecto de investigación de la Generalitat Valenciana con referencia AICO/2020/327. 


\section{INTRODUCCIÓN}

T os discursos de la gestión empresarial se han convertido en parte de _nuestro imaginario social, influyendo en la conformación de nuestras subjetividades y haciendo que, en gran medida, integremos la lógica de los modelos económicos y empresariales que se van sucediendo con el paso de los años en nuestra forma de ser y de vivir (Alonso y Fernández Rodríguez 20i3; 20i 8; Fernández Rodríguez 2007A; 2007B). En la época neoliberal, los discursos gerenciales contribuyen a la incorporación por parte de los individuos de los valores sobre los que se sustenta el modelo de sujeto neoliberal, esto es, el emprendimiento, la autogestión y la autoexigencia, entre otros (LAVAL y DARDOT 20I 3; 20I8). Dicho sistema necesita de la adhesión "voluntaria" de los sujetos para su supervivencia y expansión, consecuentemente, también precisa de la circulación de discursos que alimenten y garanticen el ejercicio de su lógica.

Mujeres y discursos gerenciales. Hacia la autogestión feminista pretende incorporar la perspectiva feminista al estudio de los discursos que se difunden a través de la literatura gerencial dirigida a mujeres. Esta es una tarea que pretende ampliar el espacio actual de reflexión en el ámbito hispanohablante, donde en muchas ocasiones la variable de género ha sido olvidada, y, sin embargo, la influencia de dichos discursos sobre la vida de las mujeres es cada vez más acuciante. A lo largo de esta obra se identifican los diferentes discursos gerenciales dirigidos a las mujeres vigentes en nuestro imaginario social, basados en la preeminencia de valores economicistas como la maximización del beneficio y el emprendimiento, al mismo tiempo que se apunta a una peligrosa conversión del movimiento feminista en un discurso de autogestión.

\section{LOS DISCURSOS DE LA GESTIÓN EMPRESARIAL DIRIGIDOS A LAS MUJERES}

El primer capítulo de esta obra titulado "Los discursos de la gestión empresarial" (1-11) muestra la evolución del discurso gerencial hacia la flexibilidad estructural y la precariedad (López Alós 20I9; MuÑoz-Rodríguez y Santos-Ortega 20I7) como mecanismo disciplinario de las subjetividades características del "nuevo espíritu del capitalismo" (Boltanski y CHIAPELlo 2002). La literatura gerencial es una de las herramientas de expansión de dicha lógica, convirtiéndose en un arma central para la profusión de discursos psicologizantes y despolitizantes. Para entender cómo afecta la lógica neoliberal a las subjetividades actuales, necesitamos comprender el neoliberalismo no solamente como una estructura socio-económica, sino atender a su dimensión prescriptiva, que afecta a la conformación de la subjetividad individual y colec- 
tiva (GaGo 20 I4; SLOBOdian 202 I; Villacañas 2020), y permite entender el neoliberalismo como una forma de gobierno, y de gobernarse a uno/a mismo/a (Barry; Osbourne y Rose 1996).

En este sentido, la literatura gerencial ha sido tradicionalmente escrita y dirigida por/para hombres, esta realidad produce una masculinización de la literatura gerencial y sus premisas que ha promovido la imagen del ejecutivo ideal masculino como una realidad falsamente neutral (Grosz i994; MerIläinen; Tienari y Valtonen 20I3). Por esta razón, el cuerpo femenino acaba siendo representado como el "otro" inferior en función de la norma masculina, es decir, como lo abnormal (KenNy y BeLl 20 i I). Sin embargo, con la progresiva incorporación de las mujeres a los cuadros directivos, se empieza a tornar patente que el sujeto protagonista de las obras no es neutral, sino sesgadamente masculino. Y esto da pie al surgimiento de la literatura gerencial dirigida específicamente a mujeres, proceso que se aborda en el segundo capítulo.

Dicho capítulo, titulado "La literatura gerencial dirigida a mujeres" (1334) nos adentra de lleno en el surgimiento de un subgénero concreto de la literatura gerencial pensado para las mujeres, tendencia que se consolidará a finales del siglo xx e inicios del xxi. Llegados a este punto, resulta esencial que nos preguntemos por los valores sobre los que se construye el modelo de mujer líder predominante en este tipo de literatura, así como las implicaciones que se derivan de ésta, tanto para una empresa igualitaria como para una sociedad justa que ponga en jaque los principios del sistema neoliberal. Y es que, en la medida en que la ideología de la gestión empresarial forma parte de los discursos económicos, políticos, tecnológicos, sociales y culturales, es un reflejo de las desigualdades de género que vertebran nuestra sociedad. Por tanto, las imágenes construidas en la literatura gerencial sobre la empresa, sus directivos y directivas; van a influir en la perpetuación de ciertas imágenes estereotípicas sobre la presencia de mujeres y hombres en la empresa, así como de ciertos modelos de liderazgo.

Partimos pues de dicho reconocimiento, y a través de nuestro análisis podremos identificar si la igualdad de género se encuentra entre las preocupaciones de la gestión empresarial que transmite la literatura gerencial, al tiempo que detectamos si los modelos de género que se construyen y se difunden a través de la misma permiten la transformación de viejas dicotomías o si las perpetúan.

\section{UNA MIRADA FEMINISTA A LOS DISCURSOS GERENCIALES}

En tercer lugar, se desarrolla "Una mirada feminista a los discursos gerenciales" (35-60), partiendo de un análisis de la literatura gerencial dirigida a mujeres. Dicho análisis adquiere sentido si comprendemos que el poder ne- 
cesita del saber y el conocimiento para perpetuarse y legitimarse (Foucault I975; 1979; I997; 2009), y que por tanto, el saber que se genera en este tipo de obras debe responder a las exigencias de algún poder. De este modo, la literatura gerencial y el saber que genera sirven para legitimar el poder neoliberal, ayudando a que los individuos integren la lógica de la autogestión, el emprendimiento y la automejora como principios rectores en sus vidas.

Mediante la Teoría de la Valoración (Christie y Martin i997; Eggins y Slade i997; Martin 2000; Martin y Rose 2003; Martin y White 2005; White 2002), se identifican las prescripciones dentro de los textos, así como el componente valorativo y normativo de los mismos. $\mathrm{Al}$ analizar las prescripciones que se lanzan hacia las mujeres en la literatura gerencial, se identifican cuatro discursos principales.

En primer lugar, los valores centrales que sirven para edificar el modelo de liderazgo femenino en dicha literatura se basan en una noción tradicional de feminidad centrada en la explotación de valores como la emoción y la empatía, el pensamiento relacional y la capacidad multitarea, la colaboración y la comunicación inclusiva. Dichos valores se traen a colación para proponer un supuesto modelo de liderazgo "diferente" para las mujeres, promoviendo la idea de la diferencia sexual entre mujeres y hombres, ahora puesta en valor por el sistema neoliberal.

En segundo lugar, encontramos el discurso sobre el emprendimiento femenino (Heizmann y Liu 2020), que promueve la autogestión del individuo sobre todo por lo que respecta al emprendimiento de negocios, sin embargo, dicha lógica se aplica también a la vida diaria de los individuos, en este caso las mujeres, poniendo en valor la proactividad y la toma de riesgos en cada una de las facetas de nuestra vida.

En tercer lugar, el discurso sobre la maternidad y la familia adquiere tintes ambiguos, es decir, no se prescribe de forma contundente una opción u otra, sino la idea de que cada mujer debe decidir qué desea hacer con su vida y autogestionarse en base a dicha decisión. Dichas prescripciones contradictorias que dejan a dicho grupo en una posición de indefensión y de autorresponsabilidad característica de las sociedades neoliberales, pero que se multiplica al interseccionar con la variable de género.

En cuarto y último lugar, está el discurso sobre el work-life balance, un discurso que en sus raíces sirve para gestionar la dicotomía público/privado. Del análisis se desprende la individualización de un problema social —el de la conciliación de la vida familiar y laboral—, que en lugar de ser resuelto por las propias profesionales mediante una selección óptima de sus futuras parejas debería formar parte de las cuestiones éticas a tratar por la política de las empresas a la vez que encontrarse en la agenda política de los sindicatos. En ningún mo- 
mento se hace referencia a una posible política de conciliación de la empresa, o a la necesidad de una gestión ética de la misma, tampoco a la urgente necesidad de reavivar la lucha feminista dentro de las organizaciones.

En este tipo de obras la lucha siempre se desarrolla a nivel individual, por tanto, sus prescripciones despolitizan a las mujeres, alejándolas de las reclamaciones de igualdad y poniéndolas en una situación de vulnerabilidad y sobreexplotación. Por esta razón, en el cuarto capítulo ("Identidades despolitizadas", 61-74) se sostiene que las premisas de dicha literatura desarticulan una visión colectiva de los problemas sociales y de la desigualdad, poniendo siempre al individuo antes que al grupo.

En esta operación tienen gran importancia el discurso de la libre elección, el tono psicologicista de las recomendaciones y el carácter aspiracional de cambio interno inscrito en las prescripciones de las obras. Todo esto se enmarca en el postfeminismo entendido como la posición discursiva que se mantiene respecto al feminismo, esto es, a través de la configuración de sus identidades y de los discursos de los que las mujeres echan mano para llevar a cabo dicha operación, se invoca al feminismo al tiempo que se le niega, tal y como seńala McRobbie (2004, 255). De esta manera se despolitiza la lucha que éstas podrían llevar a cabo para conseguir una mayor igualdad en la empresa, ya que la desigualdad se percibe como algo individual. Consecuentemente, estas obras consiguen que las mujeres individuales se hagan cargo de problemas que son colectivos, y que, como tales, deberían ser abordados desde las estructuras estatales $\mathrm{u}$ organizacionales.

Este proceso de despolitización de los sujetos debe entenderse de forma interconectada con la psicologización de la sociedad y la cultura, fenómeno complejo que tiene por objeto la gestión de las subjetividades mediante mecanismos de autocontrol. En este marco la lógica de la autoayuda y la industria de la felicidad (BÉJAR 20i 8; Cabanas e Illouz 20 i9; Ehrenreich 20 i 2; IllouZ 2010) tienen especial importancia, ya que se redefinen para asociarse inextricablemente con el éxito (también profesional). También a través de la felicidad se reformula el feminismo, que sirve al neoliberalismo en la medida en que sustituye la consecución de la justicia social por la consecución de la felicidad personal. Al mismo tiempo, estos procesos de despolitización y psicologización de las identidades deben enmarcase en un momento en que el feminismo se encuentra presente en todos los ámbitos sociales formando parte de los productos culturales. En este espacio, hemos considerado necesario reflexionar en el siguiente capítulo en torno a la emergencia de una literatura divulgativa del feminismo, que hemos dado en llamar "literatura gerencial feminista" o "literatura de autogestión feminista", debido a sus similitudes con la literatura gerencial y la de autoayuda, pero centrada en cómo ser una buena feminista. 


\section{3. ¿UN CAMINO HACIA LA AUTOGESTIÓN FEMINISTA?}

Partiendo de la despolitización y descolectivización de la presencia de las mujeres en la gerencia y la empresa producida por estas obras, el último capítulo ("El feminismo como autogestión", 75-96), nos brinda el espacio para realizar dos operaciones: señalar el auge del feminismo como autogestión y poner en valor la repolitización de los discursos feministas como herramienta de lucha frente al modelo de sujeto neoliberal que se difunde a través de los discursos gerenciales y de la neoliberalización del feminismo.

Así pues, en un marco de popularización del feminismo, emerge todo un corpus de literatura feminista accesible para el gran público en la mayor parte de librerías. Dichas obras adquieren la forma, la estética, e incluso el lenguaje propio de la literatura de autoayuda, que acaba por asemejarse también a la literatura gerencial (Medina-Vicent 2020). En esta novedosa divulgación masiva de las ideas feministas, creemos necesario señalar un riesgo, esto es, la profusión del feminismo como autogestión, el feminismo convertido en una empresa más a gestionar. Este fenómeno nos hace percatarnos de que también dicho movimiento por la igualdad se ha convertido en un espacio de interés para el neoliberalismo, y que existe el riesgo de que se convierta en una herramienta más de dicho sistema. De este modo, comprobamos que el individualismo no es incompatible con la identificación feminista, sin embargo, esto pone de relieve que la psicologización y la despolitización están también imbricándose con parte de los discursos feministas actuales. Y es que, corremos el riesgo de que el ser feminista se convierta en un aspecto más a gestionar, es decir, que se perciba el ser feminista como un modo de autogestión, reproduciendo valores que a la larga no permiten una transformación social de las estructuras, sino un cambio exclusivamente individual.

Una vez detectados estos riesgos, hemos tratado de repensar los discursos gerenciales y su poder para la despolitización y descolectivización de las identidades feministas en la gerencia, desde las bases que nos otorga la teoría feminista. Así pues, en primer lugar tratamos de rebatir el discurso sobre el liderazgo femenino predominante en dicha literatura mediante la puesta en valor de la perspectiva feminista como posible forma de aproximarse al ejercicio del liderazgo. Consideramos que esta forma de entender el liderazgo debe potenciar también otros conceptos como el de interdependencia, trabajado por Judith Butler (2015; 2020), que permite reconocer los lazos que atan a las personas, colectivizando las identidades. Otros conceptos como el de solidaridad y sororidad, tomados de las aportaciones de Amelia Valcárcel (1997) y Marcela Lagarde (2000A; 2000B), ofrecen la posibilidad de reforzar esos lazos desde el feminismo, estableciendo principios de lucha activa que se oponen a los principios neoliberales de la literatura gerencial dirigida a mujeres. 
Esta obra pretende ser una pequeña aportación para mostrarnos alerta ante los peligros de los tiempos actuales para el feminismo, que, viviendo quizás la etapa de mayor profusión del mismo en la sociedad, también enfrenta algunos de sus mayores retos. Esto es, rearticular una lucha política a nivel global, incorporar las reclamaciones de grupos muy diversos y recuperar la raíz política de su base, entre otras tareas.

\section{Conclusiones}

A lo largo de esta obra hemos trazado el camino que va de la profusión de los discursos gerenciales dirigidos a las mujeres a través de la literatura gerencial, a la conversión del feminismo en un tema de autogestión mediante la emergencia de una especie de "literatura gerencial feminista" o "literatura de autogestión feminista”. En el recorrido que va de un punto a otro, hemos podido comprobar cómo los discursos gerenciales forman parte de nuestra vida cotidiana, contribuyendo a la conversión de los individuos en empresas. En el caso específico de las mujeres, dichos discursos adquieren una serie de particularidades que acaban por perpetuar la dicotomía de género, además de la separación entre esfera pública y privada, que pasa a ser ahora una cuestión de gestión individual. Dichas particularidades desvelan el subtexto de género más profundo que opera para construir a las mujeres como empresarias de sí mismas, siendo la feminidad tradicional y el papel como madres, dos de las expectativas de comportamiento inscritas en el modelo a seguir.

Estos procesos se enmarcan en un contexto donde los discursos de la gestión empresarial se han convertido en parte de nuestro imaginario social, influyendo en la conformación de nuestras subjetividades y haciendo que, en gran medida, integremos la lógica neoliberal en nuestro accionar diario, poniendo en valor una racionalidad economicista que atiende a valores de rentabilidad y eficacia. Aquí cabe destacar el uso que se hace de la lógica de la autoayuda y de la felicidad para difundir dichos mensajes. De aquí que hablemos de "identidades despolitizadas" cuando nos referimos a los resultados de la influencia de los discursos gerenciales en las subjetividades femeninas.

Así pues, estos procesos de despolitización y psicologización de las identidades deben enmarcase en un momento en que el feminismo se encuentra presente en todos los ámbitos sociales formando parte de los productos culturales. En este espacio, lo que hemos dado en denominar como "literatura gerencial feminista" o "literatura de autogestión feminista", debido a sus similitudes con la literatura gerencial y la de autoayuda, pero centrada en cómo ser una buena feminista, es una muestra de que el feminismo se ha convertido en 
un tema de interés para el neoliberalismo, evidentemente, esto se traduce en su progresiva mercantilización. $\mathrm{Y}$ es que, en forma de manuales divulgativos, con un lenguaje muy atractivo y asequible, se reducen algunos de los principios de la teoría feminista para ser fácilmente digeridos por el gran público. Esto no tiene porqué ser negativo en un principio, aunque sí es arriesgado cuando detrás de esa simplificación se esconde la banalización de la lucha en las calles o por ejemplo, el olvido de las diferentes experiencias de las mujeres alrededor del mundo, donde la urgencia de la lucha feminista en ciertos territorios resulta apremiante.

En resumen, a lo largo de esta obra hemos desarrollado una mirada feminista a los discursos gerenciales, para acabar por advertir un creciente fenómeno que amenaza por convertir al discurso feminista en un discurso gerencial más, es decir, convertir al feminismo en un tema de mero interés individual, una dimensión más a gestionar. Así pues, al incorporar la perspectiva feminista a los discursos que se difunden a través de la literatura gerencial dirigida a mujeres, hemos podido reflexionar en torno a cómo esto afecta a la configuración de las mujeres como sujetos neoliberales, planteándonos las implicaciones éticas que tiene para la lucha feminista desde una perspectiva crítica. Y es que, en la configuración de dichas identidades, la cooptación del feminismo por parte del neoliberalismo resulta ser una operación central, que promueve la despolitización y la psicologización de las identidades, desarticulando poco a poco la raíz política de dicho movimiento. Sin embargo, en un tiempo en que todas las esferas de la vida han sido reapropiadas por los valores del neoliberalismo, un espacio de lucha como es el feminismo, no puede permitirse ser cooptado del mismo modo. Esta obra pretende ser una pequeña aportación para mostrarnos alerta ante los peligros de los tiempos actuales para el feminismo, que, viviendo quizás la etapa de mayor profusión del mismo en la sociedad, también enfrenta algunos de sus mayores retos. 


\section{REFERENCIAS BIBLIOGRÁFICAS}

Alonso, L. E. y Fernández Rodríguez, C. J. 201 3, Los discursos del presente. Un análisis de los imaginarios sociales contemporáneos, Madrid: Siglo XXI.

Alonso, L. E. y Fernández Rodríguez, C. J. 20 i 8, Poder y sacrificio. Los nuevos discursos de la empresa, Madrid: Siglo XXI.

Barry, A.; Osbourne, T. y Rose, N. 1996, Foucault and Political Reason: Liberalism, Neo-liberalism and Rationalities of Government, Chicago: University of Chicago Press.

BÉJAR, H. 201 8, Felicidad: la salvación moderna, Madrid: Tecnos.

Boltanski, L. y Chiapello, È. 2002, El nuevo espíritu del capitalismo, Madrid: Akal.

Butler, J. 20 I 5, Notes Towards a Performative Theory of Assembly, Boston: Harvard University Press.

Butler, J. 2020, Sin miedo: Formas de resistencia a la violencia de hoy, Madrid: Taurus.

Cabanas, E. e Illouz, E. 20i9, Happycracia. Cómo la ciencia y la industria de la felicidad controlan nuestras vidas, Barcelona: Paidós.

Christie, F. y Martin, J. R. I997, Genres and Institutions: Social Processes in the Workplace and School, Londres: Cassell.

Eggins, S. y Slade, D. I 997, Analysing Casual Conversation, Londres: Cassell.

Ehrenreich, B. 20 I 2, Sonrie o muere. La trampa del pensamiento positivo, Madrid: Editorial Turner.

Fernández Rodríguez, C. J. 2007A, El discurso del Management: tiempo y narración, Madrid: Centro de Investigaciones Sociológicas.

Fernández Rodríguez, C. J. 2007B, Vigilar y organizar: una introducción a los Critical Management Studies, Madrid: Siglo XXI Editores.

Foucault, M. I975, Vigilar y castigar, Buenos Aires: Siglo XXI.

Foucault, M. I979, Microfísica del poder, Madrid: Las Ediciones de La Piqueta.

Foucault, M. I997, Las palabras y las cosas: una arqueología de las ciencias humanas, Madrid: Siglo XXI.

Foucault, M. 2009, La arqueología del saber, Madrid: Siglo XXI.

GAGO, V. 20 I4, La razón neoliberal: economías barrocas y pragmática popular, Buenos Aires: Tinta Limón. https://doi.org/10.1017/CBO9781107415324.004

Grosz, E. I994, Volatile Bodies. Towards a Corporeal Feminism, Bloomington: Indiana University Press.

Heizmann, H. y Liu, H. 2020, “Bloody Wonder Woman!": Identity performances of elite women entrepreneurs on Instagram. Human Relations. https://doi. org/10.1177/0018726720979034

Illouz, E. 2010, La salvación del alma moderna, Madrid: Katz Editores. 
Kenny, K. y Bell, E. 20 i i, "Representing the Successful Managerial Body", E. Jeanes, D. Knights y P. Y. Martin (ed.), Handbook of Gender, Work and Organization, Chichester: John Wiley, 163-76.

LAGARde, M. 2000A, Claves feministas para el poderio y la autoestima de las mujeres, Managua: Puntos de Encuentro.

Lagarde, M. 2000B, Claves feministas para la autoestima de las mujeres, Madrid: Editorial Horas y Horas.

Laval, C. y Dardot, P. 2013. La nueva razón del mundo. Ensayo sobre la sociedad neoliberal, Barcelona: Gedisa.

LAval, C. y Dardot, P. 20 i 8, El ser neoliberal, Barcelona: Gedisa.

López Alós, J. 20 I9, Crítica de la razón precaria. La vida inteelctual ante la obligación de lo extrarodinario, Madrid: La Catarata.

Martin, J. R. 2000, “Beyond Exchange: Appraisal Systems in English”, S. HunsTON y G. Thompson (ed.), Evaluation in Text. Authorial Stance and the Construction of Discourse, Oxford: Oxford University Press, 142-75.

Martin, J. R. y Rose, D. 2003, Working with Discourse: Meaning Beyond the Clause, Londres: Continuum.

Martin, J. R. y White, P. R. R. 2005, The Language of Evaluation: The Appraisal in English, Nueva York: Palgrave Macmillan. https://doi. org/10.1057/9780230511910

McRobbie, A. 2004, "Post-Feminism and Popular Culture", Feminist Media Studies, 4 (3): 255-64. https://doi.org/10.1080/1468077042000309937

Medina-Vicent, M. 2020, "La literatura gerencial en tiempos de popularización del feminismo", Revista Española de Sociología, 29 (2): 383-91. https://doi. org/10.22325/fes/res.2020.21

Meriläinen, S.; Tienari, J. y Valtonen, A. 20I3, "Headhunters and the 'Ideal' Executive Body", Organization, 22 (1): 3-22. https://doi. org/10.1177/1350508413496578

Muñoz-Rodríguez, D. y Santos-Ortega, A. 2017, En las cárceles del capital humano. Nuevas precariedades y formas de subjetivación de los procesos contemporáneos de precarización, Carcaixent-Málaga: Baladre-Zambra.

Slobodian, Q. 202 I, Globalistas. El fin de los imperios y el nacimiento del neoliberalismo, Madrid: Capitán Swing.

VAlCÁrCel, A. I997, La politica de las mujeres, Madrid: Cátedra.

Villacañas, J. L. 2020, Neoliberalismo como teología politica. Habermas, Foucault, Dardot, Laval y la historia del capitalismo contemporáneo (Ned Edicio). Ulzama.

White, P. R. R. 2002, "Appraisal-the Language of Evaluation and Stance”, J. Verschueren; J.-O. Östman y J. Blommaert (ed.), Handbook of Pragmatics, Ámsterdam: John Benjamins, 1-23. 